\title{
Brain-Computer Interfaces: From Past to Future
}

\author{
Murad Sultanov* \\ Laboratory of Ecological Toxicology, National Academy of Sciences of Azerbaijan, Azerbaijan \\ *Corresponding author: Murad B. Sultanov, Laboratory of Ecological Toxicology; AI Qaraev Institute of Physiology, National \\ Academy of Sciences of Azerbaijan, Azerbaijan.
}

To Cite This Article: Murad Sultanov. Brain-Computer Interfaces: From Past to Future. Am J Biomed Sci \& Res. 2019 - 4(3). AJBSR.MS.ID.000799. DOI: 10.34297/AJBSR.2019.04.000799

Received: July 26, 2019 | Published: July 31, 2019

\begin{abstract}
More than 100 years ago, scientists were interested in the capabilities of the brain and tried to understand whether it is possible to somehow influence it. In 1875, English doctor Richard Caton managed to register a weak electric field on the surface of the brain of rabbits and monkeys. Then there was a lot of discovery and research, but only in 1950, José Manuel Rodríguez Delgado, a professor of physiology at Yale University, invented the device, which could be implanted in the brain and controlled by radio signals. Thus, began the era of neural interfaces and technologies capable of enhancing human biological capabilities. Already in 1972, a cochlear implant went on sale, which converted the sound into an electrical signal, transmitted it to the brain and actually allowed people with severe hearing impairments to hear. Moreover, in 1973, for the first time, the term "brain-computer interface" was used - a computer-based neuro interface. .
\end{abstract}

Keywords: Brain-Computer interface; Neurofeedback; Non-Invasive method; EEG

\section{Introduction}

The biofeedback and neurofeedback technology developed in the middle of the 20th century proved a simple but effective way of regulating the physiological characteristics, in particular, the frequency of cardiac contractions, the tone of blood vessels, and the electrical activity of the human brain [1]. Neuro interface - a system for the exchange of information between the human brain and an electronic device. This is a technology that allows a person to interact with the outside world based on the recording of the electrical activity of the brain is electroencephalogram (EEG). A person's desire to perform some action is reflected in EEG changes, which in turn decodes the computer. Neural interfaces are unidirectional and bidirectional. The former either receive signals from the brain or send them to him. The latter can send and receive signals simultaneously. In addition to training and entertainment programs, developers have created a large number of applications designed for professional use. As an example, the software Mind Rec by Neuro Sky, which is designed for medical, sports, ordinary psychologists and psychologists working with representatives of power structures.

How is it used? The person puts on a neuro headset, the psychologist starts the program and starts the session. During the session, the following information is monitored and stored in the computer'smemory,namely:thelevel ofconcentration, attentiveness, the raw EEG signal, in several types of visualization at a time, in the range from 0 to $70 \mathrm{~Hz}$. Signals divided by frequency ranges that make up the spectrum of the main signal. The breakdown is made on eight ranges: Delta, Theta, Low Alpha, High Alpha, Low Beta, High Beta, Low Gamma, and High Gamma. If necessary, audio and video recordings of the patient psychologist's actions are performed. Recorded material can be reviewed by seeing everything that was displayed in real time during the session. If the psychologist did not notice something immediately, then when restudying a session or a workout, he can study changes in the wave reactions of the brain, compare them with audiovisual information. This is a very valuable tool for any specialist in this field.

Brain-Computer Interface (BCI) technology for neurofeedback include from prosthetics and control systems [2] to medical diagnostics [3]. Training brainwave activity may make it easier to enter a state of alertness, or, on the other hand, make it easier to enter a relaxed state [4]. Along with this, neurofeedback is actively used in training users of systems BCI, and how practically healthy [5] and, in particular, completely paralyzed persons [6,7]. In addition to parameters for neurofeedback can be quite wide and include specificities of almost all EEG bands both low-frequency $(0.1-30 \mathrm{~Hz})$ and high-frequency (more than $30 \mathrm{~Hz}$ ) range. Together, determining the relationship of individual psychological and neurophysiological characteristics is today one of the urgent tasks of psychophysiology. There are many mobile systems, which is designed for medical, sports, ordinary psychologists and psychologists working with representatives in military service. Furthermore, increasing 
interest to the investigation of electrical activity of brain during active biomechanical actions, such as walking, running, various kinds of sports has been one of the main causative factors in the active development and application of wireless neurocomputers in industry for the calculation of electroencephalic signals and their derivatives.

\section{Conclusion}

Research into neurofeedback, including of fMRI decoding psychology state open new opportunities for dimension in biological recording, however the importunacy of the analyzing equipment makes it difficult to turn to account from data catch to provide feedback [8]. Thus, neurofeedback from lightweight wireless EEG system (Neuro Sky; Emotiv et al.) might be used to research of psychological characteristics in people, who cannot travel to a traditional EEG laboratory, for example, elderly people, who cannot tolerate laboratory conditions, for example people with autism, depression and other behavioral diseases; along with that children with $\mathrm{AD} / \mathrm{HD}$.

\section{References}

1. Gruzelier JH (2014) EEG-neurofeedback for optimising performance. III: a review of methodological and theoretical considerations. Neurosci Biobehav Rev 44: 159-182.
2. Loudin JD, Simanovskii DM, Vijayraghavan K, Sramek CK, et al. (2007) Optoelectronic retinal prosthesis: system design and performance. J Neural Eng 4(1): S72-84.

3. Fong SS, Tsang WW, Cheng YT, Ki WY, et al. (2015) Single-channel electroencephalographic recording in children with developmental coordination disorder: validity and influence of eye blink artifacts. Journal of Novel Physiotherapies.

4. Ramsay JR (2010) Nonmedication treatments for adult ADHD: Evaluating impact on daily functioning and well-being. American Psychological Association.

5. Hwang HJ, Kwon K, Im CH (2009) Neurofeedback-based motor imagery training for brain-computer interface (BCI). J Neurosci Methods 179(1): 150-156.

6. Birbaumer N, Murguialday AR, Weber C, Montoya P (2009) Neurofeedback and brain-computer interface: clinical applications. Int Rev Neurobiol 86: 107-117.

7. Hoffmann U, Vesin JM, Ebrahimi T, Diserens K (2008) An efficient P300-based brain-computer interface for disabled subjects. J Neurosci Methods 167(1): 115-125.

8. Haynes JD, Rees G (2006) Neuroimaging: decoding mental states from brain activity in humans. Nat Rev Neurosci 7(7): 523-534. 